\title{
Cervical cancer: preventable deaths of women to shape up the future
}

\author{
Deepa Gamage \\ Consultant Epidemiologist, Epidemiology Unit, Ministry of Health, Sri Lanka \\ Correspondence: deepagamage@gmail.com
}

DOI: https://doi.org/10.4038/jccpsl.v23i4.8133

Received on: 14 September 2017

Accepted on: 5 December 2017

\section{- "A woman should not die or suffer from cervical cancer when it is preventable!" -}

Cervical cancer is a preventable non-communicable disease. The Global Strategy for Women, Children and Adolescent Health 2016-2030 has identified every woman, child and adolescent in all settings to realize their rights for healthy and successful living, and acquisition of all opportunities to participate fully in shaping prosperous and sustainable societies. An objective on 'survival' has been identified to end preventable deaths by giving targets for the reduction of premature mortality from non-communicable diseases (1).

\section{Cervical cancer situation: global, regional and Sri Lankan context}

Cervical cancer severely affects female health, with high mortality and morbidity worldwide. Globally, 1.4 million suffer from cervical cancer while nearly 528,000 new cases with 266,000 deaths occur annually. Cervical cancer is ranked as the fourth cause of female cancers and the second among women aged $15-44$ years in the world. It is also the second common cause of cancer deaths causing nearly $80 \%$ of deaths in developing countries. It has been globally estimated that almost $12 \%$ of all female cancers are cervical cancers (2).

In the South East Asian Region, cancer cervix accounts for 175,000 new cases and 94,000 deaths annually (2). Incomplete data reported from some countries in the absence of population-based cancer registries, preclude portraying the true burden. In most countries, information on the national cervical cancer burden is based on hospital-based cancer registries while efforts have been made in some countries such as India in implementing population-based cancer registries in some parts (3).

Sri Lanka initiated a population-based cancer registry from 2012, as a pilot project in the district of Colombo to gather information from government and private hospitals, laboratories and death registrars with the objective of obtaining comprehensive data and thereafter scaling it up to island wide registration. In Sri Lanka, it is estimated that 7.52 million are at risk of developing cervical cancer, 1395 cases with an agestandardized rate of 11.8 , and 814 deaths (2). According to the data of National Cancer Control Programme (NCCP), a total of 850 cases of cervical cancer with an age-standardized rate of 7.4 have been identified in 2008, with an incidence rate of 8.6 per 100,000 women. Similar statistics with negligible variability have been experienced during the past few years. Cervical cancer is the second commonest female cancer and accounts for $10 \%$ of all female cancers in Sri Lanka (4). However, the true burden may be higher given the fact that data is collected only from hospital registries.

\section{Relationship of human papilloma virus and cervical cancer}

Cervical cancer is caused by human papilloma virus (HPV). More than 100 HPV types have been identified, of which nearly 40 can infect the genital area. HPV is sexually transmitted via skin to skin contact and most 
people become infected sometime during their lifetime predominantly in association with sexual activities. Most infections are asymptomatic and usually clear up, accounting for $90 \%$ within two years. Nearly $10 \%$ of persistent HPV infections with certain genotypes can progress to cervical dysplasia. If infection from cancercausing (high-risk) HPV genotypes persists over 1015 years, it would lead to pre-cancerous lesions and finally develop cervical cancer.

Human papilloma virus is an essential factor in developing cervical cancer. Nearly $99.7 \%$ of cervical cancers have been attributed to genital HPV infections. HPV genotypes which cause cancers are called 'high-risk' genotypes while the non-cancer causing genotypes are called 'low-risk' genotypes. Two high-risk HPV genotypes of 16 and 18, account for about $70 \%$ of all cervical cancers. Several cofactors have been identified contributing to the acquisition and persistence of HPV infection, such as multiple sexual partners (female commercial sex workers), early age at coitarche, giving birth at very young age, multiple pregnancies, presence of other sexually transmitted infections, weak immune system (e.g. HIV), smoking, long-term use of contraceptive pills and low socio-economic status.

Global HPV prevalence on average is estimated to be $11-12 \%$ (5-6). However, this prevalence can vary according to the type of test, sample collected, method of transport and storage as well as the behavioural patterns of the country. The HPV prevalence in South East Asian countries varies around 5-18\%, while accounting for the variation in detection methods and behavioural patterns, and estimated to be $6.6 \%$ for South Asia (7).

\section{Efforts to identify HPV related cervical cancer}

Presently, HPV detection tests are not established in Sri Lanka and therefore, no country-specific routine HPV data are available. Cytological changes in Pap (Papanicolaou) smears have been identified without adequately published data. The country-specific true picture of HPV prevalence will not be reflected without scientific methodological study findings. HPV related genital warts have been detected at the National STD/ AIDS Control Programme (NSACP) as 1782 in 2012, 1911 in 2013, 1872 in 2014 and 2005 in 2015, which provide some idea on the trend of HPV genital infection related disease conditions.
A community-based descriptive cross-sectional study has been conducted in the district of Gampaha among 2000 married women aged 20-59 years to estimate the community prevalence of HPV genital infection among married women in the country (8). The HPV-DNA was detected by PCR method (40 cycles), using GP 5+/6+ primer system at the Genetech Laboratory in Sri Lanka. The genotype identification and direct sequencing was done at the laboratory of Eton Bioscience North Carolina Branch, Research Triangle Park, NC, USA, and sequences were obtained in the form of electrograms and genotypes identified at the Genetech Laboratory with the assistance of GenBank.

The community prevalence rate of HPV infection was $3.3 \%(95 \% \mathrm{CI}=3.2,3.4)$ while the prevalence rate of HPV genotype 16 and 18 was 1.2\% (95\% $\mathrm{CI}=1.15,1.25)$. The high-risk and low-risk genotypes detected in the study are given in Table 1.

\section{Table 1. High-risk and low-risk genotypes detected in the study}

\begin{tabular}{cccc}
\hline \multicolumn{2}{c}{ High-risk } & \multicolumn{2}{c}{ Low-risk } \\
\hline Genotype & No. of cases & Genotype & No. of cases \\
\hline $\mathbf{1 6}$ & 22 & $\mathbf{4 2}$ & 7 \\
$\mathbf{1 8}$ & 2 & $\mathbf{6 2}$ & 5 \\
$\mathbf{3 1}$ & 1 & $\mathbf{6 6}$ & 4 \\
$\mathbf{3 3}$ & 1 & $\mathbf{8 1}$ & 2 \\
$\mathbf{3 5}$ & 2 & $\mathbf{8 3}$ & 1 \\
$\mathbf{4 5}$ & 1 & $\mathbf{8 7}$ & 2 \\
$\mathbf{5 1}$ & 1 & & \\
$\mathbf{5 6}$ & 4 & & \\
$\mathbf{7 3}$ & 2 & & \\
\hline
\end{tabular}

The HPV prevalence among 20-29 year age group was $3.6 \%$, with a gradual reduction of the prevalence rates observed as the age increased, while a second peak (3.7\%) was observed in the age category of 50-59 years.

Lower average monthly income of less than Sri Lankan Rupees (Rs.) 5000 (prevalence of 15.4\%), social classes of 5 a (petty traders, hawker, semi-skilled labourers) and 5b (unemployed) as categorized based 
on the classification of International Labour Organization (prevalence of 5.2\% and 9.8\%, respectively), first sexual exposure below the age of 19 years (prevalence of 5.2\%), extra-marital sexual exposure (prevalence of 8.2\%) and having more than four sexual partners (prevalence of 23\%) have shown comparatively a high prevalence of HPV.

Different categorizations of non-invasive squamous lesions of the pre-cancer stages of cervical cancer are given in Table 2. Table 2. Different categorizations of cervical
pre-cancer stages

\begin{tabular}{lcc}
\hline Traditional & $\begin{array}{c}\text { WHO } \\
\text { classification }\end{array}$ & $\begin{array}{c}\text { Bethesda } \\
\text { classification }\end{array}$ \\
\hline Mild dysplasia & CIN - I & $\begin{array}{c}\text { LSIL } \\
\text { (Low grade) }\end{array}$ \\
Moderate dysplasia & CIN - II & $\begin{array}{c}\text { HSIL } \\
\text { (High grade) }\end{array}$ \\
$\begin{array}{l}\text { Severe dysplasia } \\
\text { Carcinoma-in-situ }\end{array}$ & CIN - III & \\
\hline
\end{tabular}

All 2000 samples in the community-based HPV prevalence study were tested for Pap smear to detect cytological changes at the time of the study and identify the HPV prevalent status in different microscopic entities (Table 2). Though clinically normal, one cervical cancer case and 14 pre-cancer stages of carcinomain-situ (CIN) were detected, and their HPV status is given in Table 3.

There are several stages of cervical cancer depending on the advancement of disease (from stage I in which the lesion is confined to the cervix up to stage IV in which the lesion is spread to distant organs), and the treatment modalities vary according to the stage, such as surgery, chemotherapy, radiotherapy or a combination of treatment such as chemo-radiation.

\section{Cervical cancer prevention strategies}

Public health preventive strategies in epidemiology are described as primary prevention, secondary prevention and tertiary prevention. Implementation of preventive strategies for the entire population aiming at preventing risk factor exposures to prevent the majority is expected from primary prevention. The secondary preventive strategies are aimed at early detection and treatment while tertiary prevention would treat and rehabilitate once the particular disease/s have occurred, with lower impact on prevention (Figure 1).

Table 3. Distribution of HPV status by Pap smear result

\begin{tabular}{|c|c|c|c|c|c|c|}
\hline \multirow[t]{3}{*}{ Present Pap result } & \multirow[t]{3}{*}{ Total No. } & \multicolumn{4}{|c|}{ Status of HPV } & \multirow[t]{3}{*}{ Significance } \\
\hline & & \multicolumn{2}{|c|}{ Positive } & \multicolumn{2}{|c|}{ Negative } & \\
\hline & & No. & $\%$ & No. & $\%$ & \\
\hline Normal (reference category) & 1933 & 59 & 3.1 & 1874 & 96.9 & $\begin{array}{r}\chi^{2}=8.9 \mathrm{df}=1 ; \mathrm{p}=0.002 \\
\mathrm{OR}=3.71(95 \% \mathrm{CI}: 1.37-8.59)\end{array}$ \\
\hline CIN - I ${ }^{\mathrm{a} 1}$ & 12 & 2 & 16.7 & 10 & 83.3 & \\
\hline CIN - II a2 & 2 & 1 & 50.0 & 1 & 50.0 & \\
\hline $\mathrm{CIN}-\mathrm{III}^{\mathrm{a} 3}$ & 0 & 0 & 0.0 & 0 & 0.0 & \\
\hline Cervical malignancy ${ }^{\mathrm{a} 4}$ & 1 & 0 & 0.0 & 1 & 100.0 & \\
\hline Infective/inflammatory changes a5 & 39 & 3 & 7.7 & 36 & 92.3 & \\
\hline Endometrial cells above 40 years ${ }^{\text {a6 }}$ & 13 & 1 & 7.7 & 12 & 92.3 & \\
\hline Total & 2000 & 66 & 3.3 & 1934 & 96.7 & \\
\hline
\end{tabular}

a1-a6 were amalgamated for applying chi-square test 


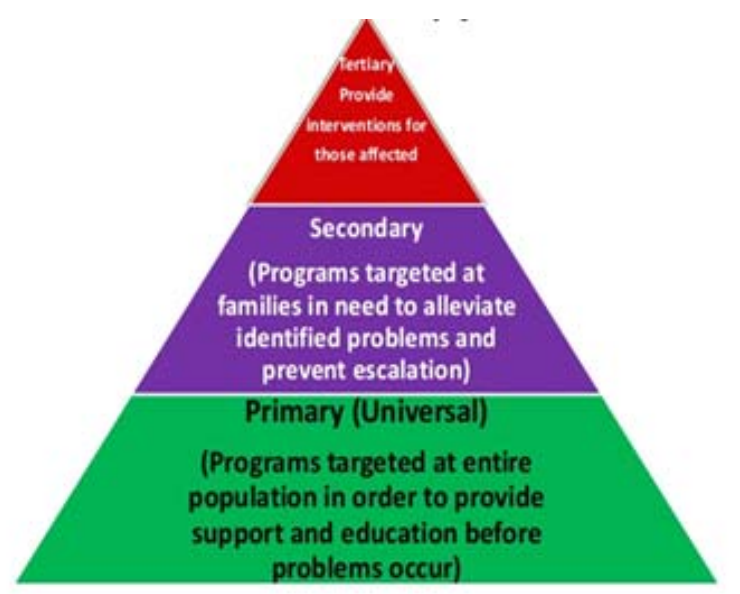

Figure 1. Public health pyramid for preventive strategies

The secondary preventive strategy of cervical cancer screening by Pap smear test was established as a national programme in Sri Lanka in 1998, to provide services through community-based well woman clinics (WWC) implemented by the Family Health Bureau. Cervical cells are collected during the procedure, preserved with alcohol and transported to laboratories with facilities for cyto-screening, to detect cervical cancer or pre-cancer stages in a well-organized manner throughout the country. In addition to this main national programme, Pap smear services are provided by the NCCP, NSACP, Cancer Institute Maharagama (presently Apeksha Hospital), gynaecological wards in government hospitals and private health care institutions, Family Planning Association and by the Cancer Society.

The current Pap smear screening is targeting the priority age group of 35 years, and the coverage of this age cohort has been around 34\% in the national programme in 2013. Recent strengthening and improvement of 35-year age cohort has been observed compared to older age cohorts. However, only around 130,000 cases per year have been screened through the national programme, including all age cohorts (9). Further, the NCCP screens an average of 2000 women annually while clinics in the NSACP screen around 1000-1500 cases per year (as per 2015 data) (10). Exact published data from government gynaecology wards and private health care institutions are not available but the contribution from these institutions would be minimal. Attention should be drawn towards the requirement of maintaining a proper data bank and triangulation of data from different institutions in 'screen and treat strategy' in secondary prevention of cervical cancer.
Special attention needs to be paid on increasing the Pap smear coverage with special consideration on the demand for resources in health care institutions, maintenance of quality, improving public awareness to create demand by beneficiaries, increasing easy accessibility and availability of quality services, adequate treatment opportunities and follow-up of screendetected cases. There are other screening methods stipulated for early detection of cervical pre-cancer under the strategy of secondary prevention. Visual inspection of the cervix has re-emerged as a screening tool for low-resource settings despite its limited specificity, since it is economical and provides immediate results. Visual inspection can be performed with acetic acid (VIA) or Lugol's iodine (VILI). The HPVDNA testing is another method in which several newer testing options are available. Current cervical cancer screening protocols typically include a combination of cervical cytology and HPV testing. The feasibility or cost assessment of newer techniques, such as HPV virus DNA detection together with Pap smear is another area to be considered in future for providing better services with improved screening coverage. Modern methods of nucleic acid-hybridization assays, signalamplification assays and nucleic-acid amplification tests are available with different testing methodologies at varying costs (11), but not yet popular in developing countries due to technology and cost implications. However, economical testing methods are available mostly in nucleic acid amplification tests and the WHO recommendations are available for combining screening methods to provide better services with high coverage for a wider population.

Cost estimation efforts have been made in the Sri Lankan scenario (8) on the government WWC Pap smear programme in 2009. Accordingly, the unit cost estimation per Pap test incurred was Rs. 308 per test. This includes all the capital and recurrent costs for Pap smear collection at clinics and Pap smear readings at the laboratory considering the rates incurred based on government purchasing prices for the estimated year (2009). Considering the annual incremental costs, and varying ingredients and novelties of techniques, the current cost incurred would vary from Rs. 300500 per test.

A prediction exercise was done as a predicted case scenario, based on the known facts on cervical cancer development combined with research study findings of the community prevalence of HPV (8). The community-based prevalence study detected cervical 
pre-cancer stages: 12 cases of CIN - 1, two cases of CIN - 2 and one case of cervical cancer out of 2000 women. Global published literature illustrates the progression proportions from pre-cancer stages to cervical cancer, which depicts $1 \%$ of CIN - 1, 1.5\% of CIN - 2 and $12 \%$ of CIN - 3 will progress into cervical cancer over time (12). On applying progression proportions to screen-detected cases in the communitybased prevalence study, the number needed to screen for prevention of one cervical cancer would be 1739 (1.15 cervical cancers progressed from 2000 women screened), but when considering women above 35 years, this number reduces to 1130 . The National Screening Programme conducts 35 year age cohort screening for cervical cancer, and the rationale for this age group is based on this fact.

The total number of women (above 35 years) needed to be screened (at this HPV prevalence status) can be calculated in prevention of the total reported cervical cancers per year, which is 850-950 cases for the country, of which nearly one million women above 35-year age group need to be screened annually to prevent the total annual case load. However, if the reported case load is under reported, the actual number of women needed to be screened (any age above 35 years) annually would be more than one million.

The minimum unit cost estimated for Pap smear was Rs. 308 and the minimum cost required to screen at community clinics $(n=1130)$ for prevention of one cervical cancer patient is Rs. 348,040. In fact, for the prevention of annual case load of 850-950 cases, this requirement is estimated to be Rs. 313 million. Pap screening is recommended to begin at the age of 21 years or start within 3 years of first sexual exposure and to stop when the woman reaches 65 years of age, optimally with recall screenings of 3-5 year intervals. However, the 'screen and treat' recommendations updated by the WHO in 2013 (13) recommended priority age group screening for limited resource settings and highlighted the importance of achieving high coverage at first screening before recalling for subsequent screenings or adding another priority age group (30-45 years). If resources permit, it is recommended to screen at five-year time intervals. Cervical cancer incidence in the country gradually increases from 35 to 70 years and peaks around 65 years (4), and if the 35-65 year age group is considered for 'recall screen and treat' procedure, it accounts for a minimum of six screening procedures per woman during her life time which accounts for minimum governmental cost of Rs. 1848 per woman. This did not consider the societal cost for women and the family as well as cost components for the treatment of screen-detected cases.

\section{HPV risk attribution of cervical cancer and cost implications on management}

Efforts on generating country-specific evidence on HPV risk attribution in developing cervical cancer, a case-control study has been conducted which included the identification of specific HPV genotypes in developing cervical cancer (8).

The study has been conducted in the Cancer Institute Maharagama among 40 cervical cancer cases and 160 compatible controls. High proportion of HPV (80\%) was detected among cervical cancer cases and the proportion of HPV detected among compatible controls (3.8\%) was similar to the HPV prevalence detected among clinically normal women in the community prevalence study (3.3\%). The most prevalent genotypes detected among cervical cancers were HPV 16 (91\%) followed by HPV 18 (6\%) and HPV 31 (3\%). Further studies done in Sri Lanka support these findings, in which $84.7 \%$ (95\% CI=76.0, 91.2) was identified among invasive cervical cancers in 2013 with the majority HPV 16 (67.3\%; 95\% CI=57.1, 76.5) followed by HPV 18 (9.2\%; 95\% CI=4.3, 16.7) (14); 93\% in 2006-7 with the majority HPV 16 and 18 (83\%) (15); and 73\% for HPV 16 and 20\% for HPV 18 in 2006 (16). This showed that the genotype distribution and prevalence among cervical cancers are similar in different geographical regions over time. Findings of the most prevalent genotypes 16 and 18 were compatible with genotypes detected in most other countries in the region and the world.

Adjusted odds ratio for HPV considering all possible risk factors for cervical cancer development has been calculated as 172 for any HPV genotype and 190 for the commonest high-risk genotypes 16 and 18. This transcribes 172 times increased risk for development of cervical cancer among those who have genital HPV infection, compared to normal women. The calculated population attributed risk (PAR\%) for high-risk genotypes 16 and 18 in developing cervical cancer was 69\% and was compatible with known HPV risk attribution (70\%) in developing cervical cancers. 
Efforts have been made to estimate the minimum cost incurred by the government in managing cervical cancer at different stages for different treatment modalities, as indicated based on scenario building technique. The unit cost referring to manage one case and cost incurred in management of cervical cancer stages by radical hysterectomy for early stages has been estimated at Rs. 13,668 and for chemo-radiation for advanced stages was Rs. 23,341. This cost estimation done in 2009 should be considered with varying annual incremental costs and changing treatment modalities over time for accurate interpretation. However, cost implications for initial investigations, subsequent follow-up costs including management costs of recurrences, managing complications, societal costs incurring to the patient and the family have not been added to these basic initial costs. This included all fixed capital costs, recurrent costs and patient management costs including services and on average five inward patient care days.

\section{Primary prevention of cervical cancer: HPV vaccination}

Prevention through primary or secondary preventive strategies could easily help women to have a brighter future by reducing morbidity and mortality and would be less expensive compared to the tertiary preventive strategies. In fact, primary preventive strategy of cervical cancer which includes the prevention of exposure to risk factors, such as behavioural changes towards safer sexual practices, avoidance of multiple partners, postponing early sexual debut and consistent condom use, and HPV vaccination would be more practical to apply for a wider population in preventing the majority.

There are three types of vaccines available globally, in which bivalent and quadrevalent HPV vaccines are given for prevention of $70 \%$ of cervical cancer providing protection for HPV high-risk genotypes 16 and 18. Age groups recommended are 9-26 years (2-dose schedule for 9-13 years and 3-dose schedule for above 13 years). Ninevalent HPV vaccine protects $90 \%$ of cervical cancers providing protection for HPV genotypes $6,11,16,18,31,33,45,52$ and 58 (17). Both bivalent and quadrevalent HPV vaccines are registered in Sri Lanka and available in the private market since 2010, and procedures are followed to include quadrevalent vaccine into the National Immunization Programme since 2017. The country has put forward steps to bear the additional costs that would be incurred to the National Immunization Programme including the supportive measures for communication strategies, including the vaccine cost (1.5 million rupees per year), for proper awareness, assurance of continuous supply chain, financial sustainability, and establishment of monitoring and evaluation.

A total package for cervical cancer prevention including all strategies of primary, secondary and tertiary prevention is a current requirement in the country. The present annual cervical cancer cases of 850-900 have missed their opportunity to be detected early through screening. While providing tertiary care facilities including rehabilitation for identified cervical cancer cases, strengthening of screening with newer screening methods, behavioural modification to prevent HPV transmission and HPV vaccination of all eligible age cohorts are requirements for the comprehensive approach of cervical cancer prevention.

A woman should not die or suffer from cervical cancer when it is preventable, as woman is a mother caring for children; family needs a surviving mother to contribute to the family bond; and a woman generates income to nurture the family. Saving lives of mothers and grand-mothers will enable generations to live together for years with quality life.

\section{References}

1. WHO. Promoting health through life-course: global strategy for women's, children's and adolescent's health 2016-2030. Geneva: World Health Organization, 2016. Available from: http://www.who.int/life-course/ partners/global-strategy/global-strategy-2016-2030/ en/index5.html.

2. GLOBOCAN 2012. Estimated cancer incidence, mortality and prevalence worldwide in 2012. France: International Agency for Research on Cancer, World Health Organization, 2013.

Available from: http://globocan.iarc.fr/.

3. Indian Council of Medical Research. Three-year Report of Population Based Cancer Registries 2009-2011. National Cancer Registry Programme. Bangalore: National Centre for Disease Informatics and Research, 2013.

Available from: http://www.icmr.nic.in/ncrp/ PBCR_Report\%202009_2011/ALL_CONTENT/ ALL_PDF/Preliminary_Pages.pdf. 
4. National Cancer Control Programme. Cancer incidence data Sri Lanka. Colombo: Ministry of Health, 2008. Available from: http://www.nccp.health.gov.lk/ index.php/publications/cancer-registries.

5. Forman D et al. Review primarily using existing peerreviewed literature and cancer estimates from GLOBOCAN 2008, 2012.

6. Bruni L, Diaz M, Castellsagué M, Ferrer E, Bosch FX, de Sanjosé S. Cervical human papillomavirus prevalence in 5 continents: meta-analysis of 1 million women with normal cytological findings. The Journal of Infectious Diseases 2010; 202(12): 1789-1799.

7. HPV Information Centre. WHO sources of data: based on systematic reviews and meta-analysis performed by ICO. International Agency for Research on Cancer, World Health Organization, 2008.

Available from: http:// www.who.int/hpv centre/_se_3. html.

8. Gamage D, Rajapaksa L, Abeysinghe MRN, De Silva A. Prevalence of carcinogenic human papilloma virus infection and burden of cervical cancer attributable to it in the district of Gampaha, Sri Lanka. Colombo: UNFPA \& Epidemiology Unit, 2012 (ISBN 978-955-837506-8).

9. Family Health Bureau. Annual Report on Family Health 2013. Colombo: Ministry of Health, 2013.

10. NSACP. Annual Report 2015 National STD/AIDS Control Programme. Colombo: Ministry of Health, 2015: 45.

11. Abreu ALP, Souza RP, Gimenes F, Consolaro MEL. A review of methods for detection of human papillomavirus infection. Virology Journal 2012; 9: 262.

12. Sellors JW \& Sankaranarayanan R. Colposcopy and Treatment of Cervical Intraepithelial Neoplasia: a Beginners’ Manual. Lyon: International Agency for Research on Cancer, 2003.

13. WHO. Comprehensive Cervical Cancer Control: a Guide to Essential Practice. Geneva: World Health Organization, 2014.

Available from: http://www.who.int/reproductivehealth/ publications/cancers/cervical-cancer-guide/en/.

14. Karunaratne K, Ihalagama H, Rohitha S, Molijn A, Gopala K, Schmidt JE, Chen J, Datta S, Mehta S. Human papillomavirus prevalence and type-distribution in women with cervical lesions: a cross-sectional study in Sri Lanka. BMC Cancer 2014; 14: 116.

15. Samarawickrema NA, Tabrizi SN, Hewavisenthi J, Leong T, Garland SM. Distribution of human papillomavirus genotypes in archival cervical tissue from women with cervical cancer in urban Sri Lanka. International Journal of Gynecology \& Obstetrics 2011; 115: 180-182.

16. De Silva R, Karunaratne K, Mendis LN, Ramesh R, Chow VTK. PCR detection and typing of human papilloma virus DNA in squamous carcinoma of cervix in a cohort of Sri Lankan women. Ceylon Medical Journal 2006; 51(3):114-117.

17. WHO. Human papillomavirus vaccines: WHO position paper May 2017. Weekly Epidemiological Record 2017; 92(19): 241-268.

Available from: http://www.who.int/immunization/ documents/positionpapers/en/. 